\title{
Effect of bilberry juice on muscle damage and inflammation in runners completing a half marathon
}

\author{
A. Lynn ${ }^{1}$, S. Garner ${ }^{1}$, N. Nelson ${ }^{1}$, T. Simper ${ }^{1}$, A. Hall ${ }^{1}$ and M. Ranchordas ${ }^{2}$ \\ ${ }^{1}$ Food and Nutrition Group, Sheffield Business School, Sheffield Hallam University S1 1 WB and ${ }^{2}$ Academy of Sport \\ and Physical Activity, Sheffield Hallam University S10 2 BP
}

Long distance running causes muscle damage, which is characterised by oxidative stress, inflammation, pain and loss of muscle force $^{(1)}$. Emerging evidence suggests that supplementation with polyphenols may protect against exercise induced muscle damage (EIMD) or accelerate recovery ${ }^{(2)}$. Bilberries are a rich source of polyphenols ${ }^{(3)}$, but no studies have reported whether bilberry consumption can influence EIMD. We conducted a single blind, randomised, placebo controlled study to determine whether bilberry juice reduced symptoms of EIMD in 21 recreationally trained runners (age 18-55 y). Participants consumed $2 \times 200 \mathrm{ml}$ of bilberry juice or energy matched control drink for $5 \mathrm{~d}$ before completing the Sheffield Half Marathon, on race day and for 2 days post-race. Measurements were taken at baseline, pre-race, post-race, $24 \mathrm{~h}$ post-race and $48 \mathrm{~h}$ post-race. The study was approved by the Ethics Committee of Sheffield Hallam University and all participants provided written informed consent.

Serum creatine kinase (CK; a marker of muscle damage) was measured using an enzyme kinetics kit (SpinReact, Girona, Spain). Serum C-reactive protein (CRP; a marker of inflammation) was measured using a commercial ELISA kit (R\&D Systems, Abingdon, UK). Delayed onset muscle soreness (DOMS) was assessed using a $200 \mathrm{~mm}$ visual analogue scale (VAS). Participants were asked to squat to a $90^{\circ}$ angle, return to a standing position and then rate their soreness on the VAS scale ${ }^{(1)}$. A mixed model ANOVA was used to analyse the data; Greenhouse-Giesser correction was used where necessary. CRP data was log transformed before analysis.

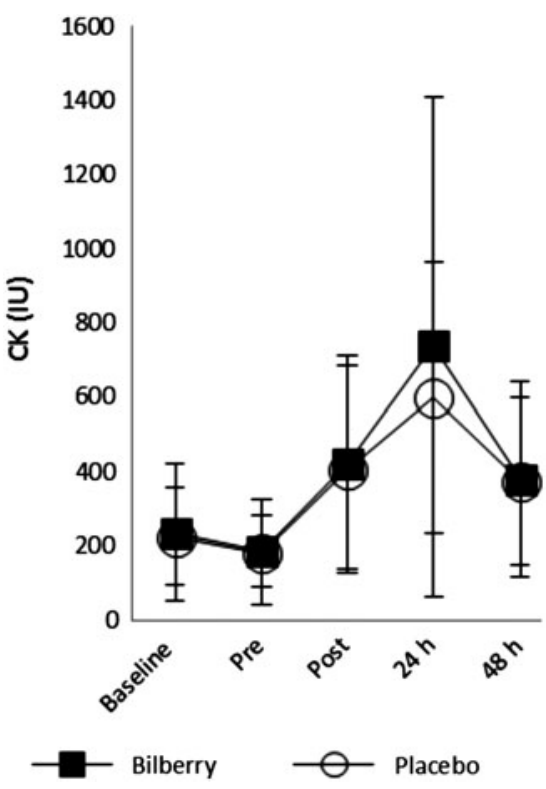

Fig 1. CK; values are mean $(\mathrm{SD})$

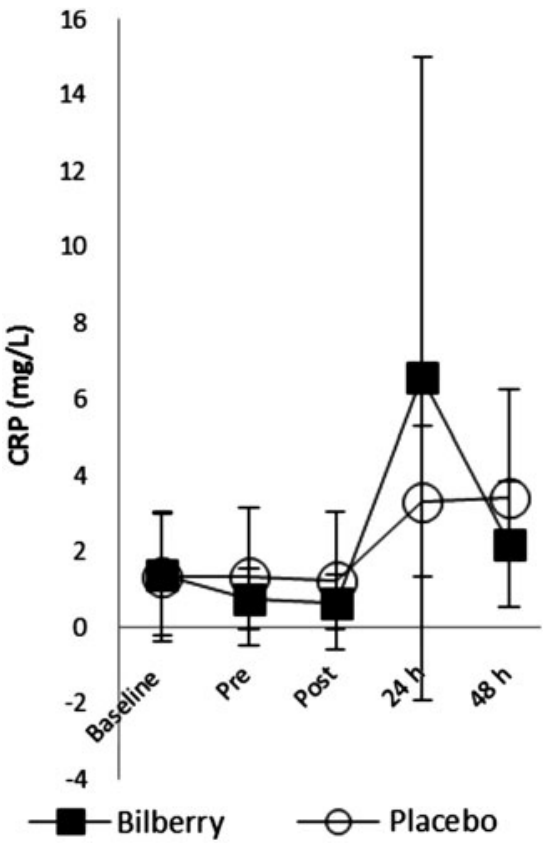

Fig 2. CRP; values are mean (SD)

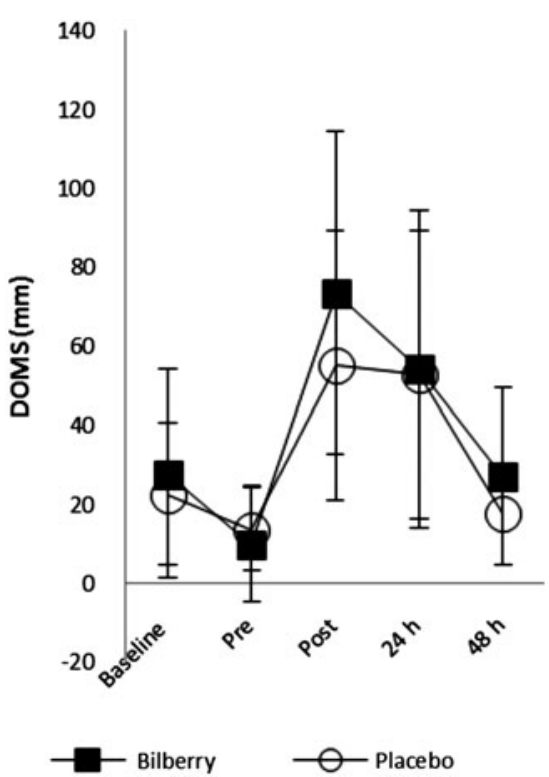

Fig 3. DOMS; values are mean (SD)

There were statistically significant main effects of time on muscle damage (Fig 1; F $(1 \cdot 50,23 \cdot 92)=12 \cdot 94 ; P<0 \cdot 001)$, inflammation $($ Fig $2 ; \mathrm{F}(1.88,31.89)=29.49 ; P<0.001)$ and DOMS (Fig 3; F $(2 \cdot 58,43 \cdot 87)=17.39 ; P<0.001)$, but no statistically significant main effects of drink type on any of the outcome measures. There were also no statistically significant interactions between drink and time. In conclusion, despite being rich in polyphenols, bilberry juice failed to attenuate the rise in muscle damage, inflammation and pain that follows a half marathon or enhance the rate of recovery.

1. Howatson G, McHugh MP, Hill JA et al. (2010) Scand J Med Sci Sports 20, 843-852.

2. Myburgh KH (2014) Sports Med 44, (Suppl 1) S57-S70.

3. Kalt W, Howell AB, MacKinnon et al. (2007) J Sci Food Agric 87, 2279-2285. 